\title{
SOBRE EL ESTABLECIMIENTO DE LA EUROPEAN ASSOCIATION OF PRIVATE INTERNATIONAL LAW (EAPIL)
}

\author{
ON THE ESTABLISHMENT OF THE EUROPEAN \\ ASSOCIATION OF PRIVATE INTERNATIONAL LAW (EAPIL)
}

\author{
European Association of Private International Law (EAPIL)
}

Recibido: 00.00.2010 / Aceptado: 00.00.2010

DOI: https://doi.org/10.20318/cdt.2020.5644

\section{Punto de partida: 50 años de europeización}

En los últimos 50 años, el desarrollo del Derecho internacional privado ha venido determinado por los esfuerzos en pro de la europeización. Desde el punto de vista histórico, esto no es nada nuevo: antes de que las normas de Derecho internacional privado se integraran en la legislación nacional durante el movimiento de codificación del siglo XIX, los estudiosos de toda Europa habían intentado durante mucho tiempo desarrollar normas europeas uniformes de determinación de la ley aplicable, en la mayoría de los casos en vano. ${ }^{1}$ Sin embargo, desde mediados del siglo XX ha surgido una red cada vez más densa de normas europeas comunes. Y desde el cambio de milenio esta red se ha desarrollado a una velocidad impresionante. De hecho, después de que los Estados miembros de la UE transfirieran la competencia legislativa en materia de derecho internacional privado a la Unión Europea en 1997, ${ }^{2}$ el legislador europeo ha adoptado un número considerable de medidas legislativas que rigen gran parte del Derecho internacional privado, tanto en el ámbito de la competencia judicial, como de la ley aplicable y el reconocimiento y ejecución de resoluciones.

En este contexto, no se puede negar el hecho de que el Derecho internacional privado se ha convertido en una disciplina verdaderamente europea. Sin embargo, hasta hace muy poco tiempo no existía ningún foro europeo que estuviera a la altura de ese desarrollo y que permitiera debatir e intercambiar opiniones sobre las normas comunes europeas. A raíz de una conferencia celebrada en Berlín en 2018, en la que se analizó críticamente la europeización del derecho internacional privado, ${ }^{3}$ se creó un Comité Directivo integrado por 14 académicos de varios países europeos ${ }^{4}$ para establecer una asociación europea dedicada al estudio y la promoción Del derecho internacional privado (europeo). Los esfuerzos del grupo pronto dieron sus frutos: en otoño de 2019, la European Association of Private International Law

\footnotetext{
${ }^{1}$ Véase Siehr, Private international law, history of, in: Jürgen Basedow, Giesela Rühl, Franco Ferrari, Pedro de Miguel Asensio (eds.), Encyclopedia of Private International Law (Edward Elgar 2017) 1390, 1392 ff.

${ }^{2}$ Hoy: Articolo 81 (1) und (2) lit. c) TFEU.

${ }^{3}$ Véase el sitio web de la conferencia: http://ceus.uni-jena.de/en/eu-pil-home así como la colección de ensayos que resultaron de la conferencia Jan von Hein, Eva-Maria Kieninger \& Giesela Rühl (eds.), How European is European Private International Law? (Intersentia 2019).

${ }^{4}$ Apostolos Anthimos (Universidad de Chipre y Colegio de Abogados Salónica, Greece); Sabine Corneloup (Universidad Paris 2 Panthéon-Assas, Francia); Gilles Cuniberti (Universidad de Luxemburgo); Morten M. Fogt (Universidad de Aarhus, Dinamarca); Pietro Franzina (Universidad Católica de Milán, Italia); Agnieszka Frackowiak-Adamska (Universidad de Wroclaw, Polonia); Jan von Hein (Universidad de Freiburg, Alemania); Thomas Kadner Graziano (Universidad de Ginebra, Suiza); Eva-Maria Kieninger (Universidad de Würzburg, Alemania); Johan Meeusen (Universidad de Amberes, Bélgica; Pedro de Miguel Asensio (Universidad Complutense de Madrid, España); Dário Moura Vicente (Universidad de Lisboa, Portugal); Marta Requejo Isidro (Instituto Max Planck de Luxemburgo); Giesela Rühl (Universidad de Jena, a partir de 1 de octubre de 2020: Universidad Humboldt de Berlin, Alemania).
} 
(EAPIL) vio la luz y se constituyó como una asociación con sede en Luxemburgo (Association sans but lucratif). ${ }^{5}$ En diciembre de 2019 se anunció oficialmente su creación ${ }^{6}$ y se puso en marcha el sitio web de la Asociación. ${ }^{7}$

\section{Objetivos y actividades de la Asociación}

La recién fundada Asociación se considera un foro para fomentar el estudio y el desarrollo del Derecho internacional privado (europeo). Sus objetivos son: 1) poner en contacto a académicos y profesionales (europeos), 2) trabajar en pro de un discurso paneuropeo que trascienda las fronteras lingüísticas y nacionales y 3 ) tender puentes a las comunidades de derecho internacional privado de los Estados no miembros de la UE, así como de los países no europeos. Persigue estos objetivos mediante diversas actividades, entre las que se destacarán tres aquí: la organización de las conferencias EAPIL, los grupos de trabajo EAPIL y el blog EAPIL.

\section{Conferencias EAPIL}

Las Conferencias EAPIL tienen por objeto reunir a los miembros de la Asociación al menos cada dos años. Siguiendo el modelo de la conferencia de Berlín de 2018, están destinadas a proporcionar una oportunidad para el intercambio, el debate y la creación de redes.

La primera Conferencia EAPIL tendrá lugar del 27 al 29 de mayo de 2021 en la Universidad de Aarhus, en Dinamarca, ${ }^{8}$ y en ella se abordarán los desafíos y problemas actuales del Derecho internacional privado. ${ }^{9}$ En particular, invitará a debatir si las normas actuales de derecho internacional privado son capaces de hacer frente a los desafíos de la digitalización y a los problemas que plantea la creciente fragmentación de las fuentes jurídicas. Entre los oradores figuran académicos de diversos países de Europa.$^{10}$ También intervendrán representantes de la Comisión Europea y del Tribunal de Justicia de la Unión Europea.

\section{Grupos de trabajo EAPIL}

Los grupos de trabajo se reúnen independientemente de las conferencias y se ocupan de cuestiones y problemas seleccionados de Derecho internacional privado (europeo) durante un período de tiempo. Tienen por objeto impulsar el desarrollo ulterior de la normativa pertinente y, en particular, apoyar la labor del legislador europeo. Las reflexiones realizadas por los Grupos de Trabajo se traducen en instrumentos (como documentos de posición o proyectos legislativos), que luego se someten a la aprobación de la Asamblea General de la Asociación.

Hasta ahora, un Grupo de Trabajo ya ha comenzado su labor. Su objetivo es examinar la viabilidad de un "Código Europeo de Derecho Internacional Privado" y presentar propuestas para su diseño.

\footnotetext{
${ }^{5}$ Registre de Commerce et de Sociétés No. F12434, L190177944.

${ }^{6} \mathrm{http}: / /$ conflictoflaws.net/2019/european-association-of-private-international-law-eapil/.

${ }^{7} \mathrm{https}$ ://eapil.org.

${ }^{8}$ La conferencia estaba originalmente programada para tener lugar del 14 al 16 de mayo de 2020. Sin embargo, debido a la situación del virus de la Corona, tuvo que ser pospuesta.

${ }^{9}$ Vea para detalles el sitio web de la conferenciahttps://law.au.dk/forskning/konferencer/eapil2020/.

${ }^{10}$ Peter Arnt Nielsen (Universidad de Copenhague, Dinamarca), Marie-Élodie Ancel (Universidad Paris-Est Créteil Val de Varne, Francia); Matthias Lehmann (Universidad de Bonn, Alemania, a partir del $1^{\circ}$ de octubre de 2020: Universidad de Viena, Austria) Burcu Yüksel Ripley (Universidad de Aberdeen, Reino Unido); Burkhard Hess (Max Planck Institute Luxembourg), Francisco Garcímartín Alférez (Universidad Autónoma de Madrid, España), Thalia Kruger (Universidad de Amberes, Bélgica); Gian Paolo Romano (Universidad de Ginebra, Suiza); Ralf Michaels (Instituto Max Planck de Hamburgo, Alemania); Marta Pertégas Sender (Universidad de Maastricht, Países Bajos), Haris Pamboukis (Universidad de Atenas, Grecia).
} 


\section{Blog EAPIL}

Una tercera actividad de la Asociación que merece ser mencionada es el Blog EAPIL. ${ }^{11}$ Está dirigido por siete editores ${ }^{12}$ y proporciona información regular sobre las actividades de la Asociación. Además, el blog EAPIL también se centra en el derecho internacional privado (europeo) como tal e informa sobre los últimos acontecimientos, a saber, decisiones judiciales, publicaciones y eventos académicos. Asimismo, constituye un foro permanente - virtual - para el intercambio de información sobre cuestiones de Derecho internacional privado (europeo).

\section{Organización y gestión de la Asociación}

La Asociación está dirigida por un Consejo de Administración y un Consejo Científico. La Junta se encarga de los asuntos cotidianos de la Asociación, mientras que el Consejo Científico se encarga de organizar las actividades académicas. La Junta está integrada actualmente por cinco miembros de Alemania, Dinamarca, Grecia, Italia y Luxemburgo..$^{13}$ El Consejo Científico está compuesto por los 14 académicos que participaron en la fundación de la Asociación. Está prevista una ampliación a 20 miembros, que se llevará a cabo durante la primera Conferencia de la EAPIL.

La Junta y el Consejo Científico son elegidos por la Asamblea General. La Asamblea General está compuesta por todos los miembros y decide sobre todos los asuntos que afectan significativamente a la existencia y al éxito a largo plazo de la Asociación. Se reúne cada dos años en el marco de las conferencias de la AAPIL.

\section{Afiliación y admisión a la Asociación}

Pueden ser miembros de la Asociación todas las personas e instituciones interesadas en el Derecho internacional privado (europeo) y que deseen contribuir a su desarrollo ulterior. La nacionalidad y la residencia son irrelevantes. Las personas e instituciones ubicadas fuera de Europa son bienvenidas a unirse.

Los detalles sobre las diversas formas de afiliación y el procedimiento de admisión pueden encontrarse en los Estatutos de la Asociación, así como en su sitio web. ${ }^{14}$ Las solicitudes de admisión pueden ser presentadas a través de la página web de la Asociación de manera muy sencilla. ${ }^{15}$

\section{Perspectivas: hacia un discurso verdaderamente europeo}

La fundación de la Asociación Europea de Derecho Internacional Privado rinde tributo a la constatación de que el Derecho internacional privado se ha convertido en una disciplina europea en los últimos años. Por consiguiente, en su calidad de foro europeo general, la Asociación desea servir de punto de contacto para los colegas de toda Europa y contribuir a la elaboración de un auténtico discurso europeo sobre el Derecho internacional privado. Que haya una demanda de ese discurso queda demostrado no solo por el éxito de la Conferencia de Berlín de 2018, sino también por la gran popularidad que ha experimentado la Asociación en los últimos meses: desde su fundación en diciembre de 2019, más

\footnotetext{
${ }^{11} \mathrm{https}$ //eapil.org/blog/.

${ }^{12}$ Los editores del blog son Apostolos Anthimos, Gilles Cuniberti, Pietro Franzina, Agnieszka Frackowiak-Adamska y Marta Requejo Isidro (véase nota 4), así como Marion Ho-Dac (Universidad de Valenciennes, Francia) y Matthias Lehmann (Universidad de Bonn, Alemania; a partir del 1 de octubre de 2020: Universidad de Viena, Austria).

${ }^{13}$ Gilles Cuniberti (Presidente); Morten M. Fogt (Vice-Presidente); Giesela Rühl (Secretaria General); Pietro Franzina (Secretario General Adjunto); Apostolos Anthimos (Tesorero).

${ }^{14} \mathrm{https} / /$ eapil.org/becoming-a-member/.

${ }^{15} \mathrm{https} / /$ eapil.org/eapil-membership-form/.
} 
de 160 juristas -académicos y profesionales- procedentes de más de 30 países se han adherido ya a la Asociación. Por lo tanto, esperamos con alegría y entusiasmo los próximos años.

Para consultas acerca de la Asociación, sus miembros y actividades, puede contactarse con su Secretaria General, Giesela Rühl, en secretary.generaly@eapil.org.

En nombre de la European Association of Private International Law:

Gilles Cuniberti, Presidente Morten M. Fogt, Vice-Presidente Giesela Rühl, Secretaria General Pietro Franzina, Secretario General Adjunto Apostolos Anthimos, Tesorero 